TAIWANESE JOURNAL OF MATHEMATICS

Vol. 19, No. 1, pp. 211-220, February 2015

DOI: $10.11650 /$ tjm.19.2015.4315

This paper is available online at http://journal.taiwanmathsoc.org.tw

\title{
EXTENSION FUNCTORS OF LOCAL COHOMOLOGY MODULES AND SERRE CATEGORIES OF MODULES
}

\author{
Nemat Abazari and Kamal Bahmanpour*
}

\begin{abstract}
Let $(R, m)$ be a complete Noetherian local ring, $I$ a proper ideal of $\mathrm{R}$ and $M, N$ two finitely generated R-modules such that $\operatorname{Supp}(N) \subseteq V(I)$. Let $t \geq 0$ be an integer such that for each $0 \leq i \leq t$, the $R$-module $H_{I}^{i}(M)$ is in dimension $<n$. Then we show that each element $L$ of the set $\mathfrak{J}$, which is defined as:

$$
\begin{gathered}
\left\{\operatorname{Ext}_{R}^{j}\left(N, H_{I}^{i}(M)\right): j \geq 0 \text { and } 0 \leq i \leq t\right\} \\
\cup\left\{\operatorname{Hom}_{R}\left(N, H_{I}^{t+1}(M)\right), \operatorname{Ext}_{R}^{1}\left(N, H_{I}^{t+1}(M)\right)\right\}
\end{gathered}
$$

is in dimension $<n-2$ and so as a consequence, it follows that the set

$$
\operatorname{Ass}_{R}(L) \cap\{\mathfrak{p} \in \operatorname{Spec}(R): \operatorname{dim}(R / \mathfrak{p}) \geq n-2\}
$$

is finite. In particular, the set

$$
\operatorname{Ass}_{R}\left(\oplus_{i=0}^{t+1} H_{I}^{i}(R)\right) \cap\{\mathfrak{p} \in \operatorname{Spec}(R): \operatorname{dim}(R / \mathfrak{p}) \geq n-2\}
$$

is finite. Also, as an immediately consequence of this result it follows that the $R$-modules $\operatorname{Ext}_{R}^{j}\left(N, H_{I}^{i}(M)\right)$ are in dimension $<n-1$, for all integers $i, j \geq$ 0 , whenever $\operatorname{dim}(M / I M)=n$. These results generalizes the main results of Huneke- Koh [17], Delfino [10], Chiriacescu [9], Asadollahi-Naghipour [1], Quy [18], Brodmann-Lashgari [7], Bahmanpour-Naghipour [5] and Bahmanpour et al. [6] in the case of complete local rings.
\end{abstract}

\section{INTRODUCTION}

Let $R$ be a commutative Noetherian ring (with identity) and $I$ an ideal of $R$. The local cohomology modules $H_{I}^{i}(M), i=0,1,2, \ldots$, of an $R$-module $M$ with respect

Received January 14, 2014, accepted May 21, 2014.

Communicated by Bernd Ulrich.

2010 Mathematics Subject Classification: 13D45, 14B15, 13 E05.

Key words and phrases: Associated prime ideal, Cofinite module, Complete local ring, Krull dimension, Local cohomology.

*Corresponding author. 
to $I$, with support in $V(I)$, were introduced by A. Grothendieck in $[14,15]$. The local cohomology module $H_{I}^{i}(M)$ is defined as:

$$
H_{I}^{i}(M)=\underset{n \geq 1}{\lim _{n \geq 1}} \operatorname{Ext}_{R}^{i}\left(R / I^{n}, M\right) .
$$

The module $H_{I}^{i}(M)$ has both algebraic and geometric aspects. In particular, there are several applications for this modules in algebraic geometry, for some applications of this type see [8]. It is well-known that in general the local cohomology module $H_{I}^{i}(M)$ is not a finitely generated $R$-module, even when $M$ is finitely generated. But for each $i \geq 0$ and each finitely generated module over an arbitrary Noetherian local ring $(R, \mathfrak{m})$ the $R$-module $H_{\mathfrak{m}}^{i}(M)$ is Artinian and hence the $R$-module $\operatorname{Hom}_{R}\left(R / \mathfrak{m}, H_{\mathfrak{m}}^{i}(M)\right)$ is finitely generated. This was lead to a conjecture from A. Grothendieck in [15], that for any ideal $I$ of a Noetherian ring $R$ and any finitely generated $R$-module $M$, the module $\operatorname{Hom}_{R}\left(R / I, H_{I}^{i}(M)\right)$ is finitely generated. This conjecture is not true in general and the first counterexample for the Grothendieck's conjecture was found by Hartshorne (see [16] for details and proof). But we know that, for any Noetherian ring of dimension $d \geq 3$ there are an ideal $I$ of $R$ and a finitely generated $R$-module $M$, such that the module $\operatorname{Hom}_{R}\left(R / I, H_{I}^{i}(M)\right)$ is not finitely generated (See [4, Theorem 3.9]). But, he defined an $R$-module $M$ to be $I$-cofinite if $\operatorname{Supp}(M) \subseteq V(I)$ and $\operatorname{Ext}_{R}^{j}(R / I, M)$ is finitely generated for all $j$ and asked:

For which rings $R$ and ideals $I$ are the modules $H_{I}^{i}(M) I$-cofinite for all $i$ and all finitely generated modules $M$ ?

Concerning this question, himself in [16] showed that for the case where dim $(M / I M)=2$ the answer is negative in general. But, with respect to this question, in the case where $\operatorname{dim}(M / I M) \leq 1$ there are several interesting papers (See for example, [9], [16, Corollary 7.7], [17, Theorem 4.1], [10], [11, Theorem 1], [22, Theorem 1.1], [5]). In [6], K. Bahmanpour et al., introduced the new notation of the $n$-th finiteness dimension $f_{I}^{n}(M)$, for all $n \in \mathbb{N}_{0}$. More precisely, they used the following notation:

$$
f_{I}^{n}(M):=\inf \left\{f_{I R_{\mathfrak{p}}}\left(M_{\mathfrak{p}}\right) \mid \mathfrak{p} \in \operatorname{Supp}(M / I M) \text { and } \operatorname{dim}(R / \mathfrak{p}) \geq n\right\},
$$

where, for each ideal $J$ of $R$ and each finitely generated $R$-module $N$, the finiteness dimension $f_{J}(N)$ of $N$ relative to $J$ is defined as:

$$
f_{J}(N):=\inf \left\{i \in \mathbb{N}_{0} \mid H_{J}^{i}(N) \text { is not finitely generated. }\right\}
$$

(Note that $f_{I}^{0}(M)=f_{I}(M)$.) Also they proved that, when $M$ is a finitely generated $R$-module, then the $R$-module $H_{I}^{i}(M)$ is $I$-cofinite for all $i<f_{I}^{2}(M)$ and in the case that $R$ is complete local the $R$-module $H_{I}^{i}(M)$ is $I$-weakly cofinite for all $i<f_{I}^{3}(M)$. Recall that an $R$-module $M$ is said to be $I$-weakly cofinite if $\operatorname{Supp}(M) \subseteq V(I)$ and $\operatorname{Ext}_{R}^{j}(R / I, M)$ is weakly Laskerian for all $j$. Also recall that an $R$-module $M$ is said 
to be weakly Laskerian or skinny if any homomorphic image of $M$ has finitely many associated primes. In [2] K. Bahmanpour has shown that over a Noetherian ring an $R$-module $M$ is weakly Laskerian if and only if the set $\operatorname{Supp}(M / N)$ is finite, for some finitely generated submodule $N$ of $M$. In particular, $\operatorname{dim}(M / N) \leq 1$. Moreover, in [1], Asadollahi and Naghipour introduced the notation of the $R$-modules in dimension $<n$, for all $n \in \mathbb{N}_{0}$. More precisely, by them definition, an $R$-module $M$ is said to be in dimension $<n$ if and only if $\operatorname{dim}(M / N)<n$ for some finitely generated submodule $N$ of $M$. (Note that here we define the dimension of the zero $R$-module equal to -1 . So each finitely generated $R$-module is in dimension $<0$. In particular, an $R$-module $T$ with support in $V(I)$ is $I$-cofinite if and only if the $R$-modules $\operatorname{Ext}_{R}^{j}(R / I, T)$ are in dimension $<0$, for all integers $j \geq 0$ ). Moreover, they showed that if $R$ is complete and local then for any finitely generated $R$-module $M$ there is another description of $f_{I}^{n}(M)$ as follows:

$$
f_{I}^{n}(M)=\inf \left\{0 \leq i \in \mathbb{Z} \text { : the } R \text {-module } H_{I}^{i}(M) \text { is not in dimension }<n\right\} .
$$

Now, let $(R, \mathfrak{m})$ be a complete local ring. Then in view of [6, Theorem 3.2], the $R$-modules $H_{I}^{i}(M)$ are $I$-cofinite, for all integers $i$ with $0 \leq i<f_{I}^{2}(M)$. Therefore, the $R$-modules $\operatorname{Ext}_{R}^{j}\left(R / I, H_{I}^{i}(M)\right)$ are finitely generated and so are in dimension $<0$, for all integers $0 \leq i<f_{I}^{2}(M)$ and $j \geq 0$. Now, it is natural that we ask:

Question: If $(R, \mathfrak{m})$ is a complete local ring, then for all $i<f_{I}^{n}(M)$ and all $j \geq 0$, are the $R$-modules $\operatorname{Ext}_{R}^{j}\left(R / I, H_{I}^{i}(M)\right)$ in dimension $<n-2$ ?

The main purpose of this paper is to prepare an affirmative answer to this question in general. Moreover, we shall give some applications of this result concerning the finiteness of special sets of associated prime ideals of the local cohomology modules. Throughout this paper, $R$ will always be a commutative Noetherian ring with nonzero identity and $I$ will be an ideal of $R$. For each $R$-module $L$, we denote by $\operatorname{Ass}_{R}(L)$ the set of associated prime ideals of $L$. Also, for any ideal $\mathfrak{a}$ of $R$, we denote $\{\mathfrak{p} \in \operatorname{Spec} R: \mathfrak{p} \supseteq \mathfrak{a}\}$ by $V(\mathfrak{a})$. For any unexplained notation and terminology we refer the reader to [8] and [21].

\section{The Results}

Before bringing our results, let $R$ be a Noetherian ring, $I$ an ideal of $R$ and $n \in \mathbb{N}_{0}$. In this paper, the symbol $\mathcal{C}_{<n}(R)$ denotes the category of all $R$-modules in dimension $<n$ and the symbol $\mathcal{C}_{<n}(I, R)$ denotes the category of all $R$-modules $T$, such that $\operatorname{Ext}_{R}^{j}(R / I, T)$ is in $\mathcal{C}_{<n}(R)$, for all $j \geq 0$. The following three lemmas, which some of them are suitable generalizations of some well-known results to Serre subcategories of the category of $R$-modules, are needed in this paper. 
Lemma 2.1. Let $R$ be a Noetherian ring, and $n \in \mathbb{N}_{0}$. Then $\mathcal{C}_{<n}(R)$ is closed under taking submodules, quotients and extensions, i.e., it is a Serre subcategory of the category of $R$-modules. In particular, for any ideal $I$ of $R, \mathcal{C}_{<n}(R)$ is a subcategory of the $\mathcal{C}_{<n}(I, R)$.

Proof. For first statement, use the method applied in the proof of [3, Lemma 2.1]. For second statement, consider a free resolution of finitely generated free modules for the $R$-module $R / I$ and use the first part of the assertion.

Lemma 2.2. Let $R$ be a Noetherian ring and $I$ an ideal of $R$ and $\mathfrak{S}$ be a Serre subcategory of the category of $R$-modules. Then, for any $R$-module $T$ and any integer $t \in \mathbb{N}_{0}$, the following conditions are equivalent:

(i) $\operatorname{Ext}_{R}^{i}(R / I, T)$ is in $\mathfrak{S}$, for all $0 \leq i \leq t$.

(ii) For any finitely generated R-module $N$ with support in $V(I), \operatorname{Ext}_{R}^{i}(N, T)$ is in $\mathfrak{S}$, for all $0 \leq i \leq t$.

Proof. For proving the assertion we use the proof of [19, Lemma 1].

Lemma 2.3. Let $R$ be a Noetherian ring and $I$ an ideal of $R$ and $\mathfrak{S}$ be a Serre subcategory of the category of $R$-modules. Let $s$ be a non-negative integer and let $M$ be an R-module such that $\operatorname{Ext}_{R}^{s}(R / I, M)$ (resp. $\left.\operatorname{Ext}_{R}^{s+1}(R / I, M)\right)$ is in $\mathfrak{S}$. If $\operatorname{Ext}_{R}^{j}\left(R / I, H_{I}^{i}(M)\right)$ is in $\mathfrak{S}$, for all $0 \leq i<s$ and all $j \geq 0$, then the R-module $\operatorname{Hom}_{R}\left(R / I, H_{I}^{s}(M)\right)\left(\right.$ resp. $\operatorname{Ext}_{R}^{1}\left(R / I, H_{I}^{s}(M)\right)$ is in $\mathfrak{S}$.)

Proof. Use the proof of [12, Theorem 2.1] (resp. [13, Theorem A]).

The following well known lemma plays key role in the proof of our main result.

Lemma 2.4. Let $R$ be a Noetherian ring and $I$ an ideal of $R$. Let $M$ be a finitely generated $R$-module such that $\left(H_{I}^{i}(M)\right)_{\mathfrak{p}}$ is a finitely generated $R_{\mathfrak{p}}$-module for all $i<t$ and for all $\mathfrak{p} \in \operatorname{Supp} M / I M$ with $\operatorname{dim} R / \mathfrak{p}>1$, where $t$ is a non-negative integer. Then $\operatorname{Hom}_{R}\left(R / I, H_{I}^{t}(M)\right)$ is a finitely generated $R$-module and $H_{I}^{i}(M)$ is a I-cofinite R-module, for all $i<t$.

Proof. See [6, Proposition 3.1].

The following theorem is crucial for the proof of the main result.

Theorem 2.5. Let $(R, \mathfrak{m})$ be a Noetherian complete local ring, $I$ an ideal of $R$, $t \geq 0$ and $n \geq 2$ be two integers and $M$ be a finitely generated $R$-module such that for each $0 \leq i \leq t$ the $R$-module $H_{I}^{i}(M)$ is in $\mathcal{C}_{<n}(R)$. Then the following statements hold:

(i) for each $0 \leq i \leq t$ the $R$-module $H_{I}^{i}(M)$ is in $\mathcal{C}_{<n-2}(I, R)$. 
(ii) The R-modules $\operatorname{Hom}_{R}\left(R / I, H_{I}^{t+1}(M)\right)$ and $\operatorname{Ext}_{R}^{1}\left(R / I, H_{I}^{t+1}(M)\right)$ are in $\mathcal{C}_{<n-2}(R)$.

\section{Proof.}

(i) If $n=2$ then the assertion follows from [6, Theorem 3.2] and if $n=3$ then the assertion follows from [6, Theorem 3.9] and [2, Theorem 3.3]. But, for $n \geq 4$, by hypothesis for each $0 \leq i \leq t, H_{I}^{i}(M)$ is in $\mathcal{C}_{<n}(R)$ and so it follows from Lemma 2.1 that $H_{I}^{i}(M)$ is in $\mathcal{C}_{<n}(I, R)$. Therefore, by the definition for each $0 \leq i \leq t$ and each $j \in \mathbb{N}_{0}$ there exists a finitely generated submodule $N_{i, j} \subseteq \operatorname{Ext}_{R}^{j}\left(R / I, H_{I}^{i}(M)\right)$ such that $\operatorname{dim}\left(\operatorname{Ext}_{R}^{j}\left(R / I, H_{I}^{i}(M)\right) / N_{i, j}\right)<n$. First we show that for each $0 \leq i \leq t$ the $R$-module $H_{I}^{i}(M)$ is in $\mathcal{C}_{<n-1}(I, R)$. To do this, as by the definition

$$
H_{I}^{i}(M) \cong \underset{n \geq 1}{\lim _{n \rightarrow 1}} \operatorname{Ext}_{R}^{i}\left(R / I^{n}, M\right)
$$

it follows that

$$
\operatorname{Ext}_{R}^{j}\left(R / I, H_{I}^{i}(M)\right) \cong \underset{n \geq 1}{\lim _{n \rightarrow 1}} \operatorname{Ext}_{R}^{j}\left(R / I, \operatorname{Ext}_{R}^{i}\left(R / I^{n}, M\right)\right),
$$

and so the $R$-module $\operatorname{Ext}_{R}^{j}\left(R / I, H_{I}^{i}(M)\right)$ has a countable generator set. In particular, there is a chain

$$
L_{1} \subseteq L_{2} \subseteq \cdots
$$

of finitely generated submodules of the $R$-module $\operatorname{Ext}_{R}^{j}\left(R / I, H_{I}^{i}(M)\right)$ such that

$$
\operatorname{Ext}_{R}^{j}\left(R / I, H_{I}^{i}(M)\right)=\bigcup_{n=1}^{\infty} L_{n} .
$$

So, for each $0 \leq i \leq t$ and each $j \in \mathbb{N}_{0}$, we have

$$
\operatorname{Ext}_{R}^{j}\left(R / I, H_{I}^{i}(M)\right) / N_{i, j}=\bigcup_{n=1}^{\infty}\left(L_{n}+N_{i, j}\right) / N_{i, j} .
$$

Therefore, the set

$$
\operatorname{Ass}_{R} \operatorname{Ext}_{R}^{j}\left(R / I, H_{I}^{i}(M)\right) / N_{i, j}=\bigcup_{n=1}^{\infty} \operatorname{Ass}_{R}\left(L_{n}+N_{i, j}\right) / N_{i, j}
$$

is countable. Consequently, the set

$$
\mathcal{A}:=\bigcup_{i=0}^{t} \bigcup_{j \geq 0} \operatorname{Ass}_{R} \operatorname{Ext}_{R}^{j}\left(R / I, H_{I}^{i}(M)\right) / N_{i, j}
$$


is countable. Now, let

$B:=\left\{\mathfrak{p} \in \operatorname{Supp}\left(\oplus_{i=1}^{t} \oplus_{j=0}^{\infty} \operatorname{Ext}_{R}^{j}\left(R / I, H_{I}^{i}(M)\right) / N_{i, j}\right): \operatorname{dim}(R / \mathfrak{p})=n-1\right\}$.

Then, it is clear that $B \subseteq \operatorname{Supp}\left(\oplus_{i=1}^{t} H_{I}^{i}(M)\right)$. Moreover, it is easy to see that each element of the set $B_{i, j}:=B \cap \operatorname{Supp}\left(\operatorname{Ext}_{R}^{j}\left(R / I, H_{I}^{i}(M)\right) / N_{i, j}\right)$ is a minimal element of $\operatorname{Supp}\left(\operatorname{Ext}_{R}^{j}\left(R / I, H_{I}^{i}(M)\right) / N_{i, j}\right)$ and so is an associated prime of $\operatorname{Ext}_{R}^{j}\left(R / I, H_{I}^{i}(M)\right) / N_{i, j}$. Therefore,

$$
B_{i, j} \subseteq \operatorname{Ass}_{R}\left(\operatorname{Ext}_{R}^{j}\left(R / I, H_{I}^{i}(M)\right) / N_{i, j}\right),
$$

for each $0 \leq i \leq t$ and each $j \in \mathbb{N}_{0}$.

Now, first we claim that $B_{i, j}$ is a finite set, for each $0 \leq i \leq t$ and each $j \in \mathbb{N}_{0}$. Because, if $B_{i, j}$ is infinite, then there exists a countably infinite subset $\left\{\mathfrak{q}_{k}\right\}_{k=1}^{\infty}$ of $B_{i, j}$. Let $S$ be the multiplicatively closed subset $R \backslash \bigcup_{k=1}^{\infty} \mathfrak{q}_{k}$. Then, it easily follows from [20, Lemma 3.2], and Lemma 4, that $S^{-1}\left(\operatorname{Ext}_{R}^{j}\left(R / I, H_{I}^{i}(M)\right) / N_{i, j}\right)$ is a finitely generated $S^{-1} R$-module, and so $\operatorname{Ass}_{S^{-1} R} S^{-1}\left(\operatorname{Ext}_{R}^{j}\left(R / I, H_{I}^{i}(M)\right)\right.$ $\left./ N_{i, j}\right)$ is a finite set. But $S^{-1} \mathfrak{q}_{k} \in \operatorname{Ass}_{S^{-1} R} S^{-1}\left(\operatorname{Ext}_{R}^{j}\left(R / I, H_{I}^{i}(M)\right) / N_{i, j}\right)$ for all $k=1,2, \ldots$, which is a contradiction. Now, if there exists an integer $i$ with $0 \leq i \leq t$ such that $H_{I}^{i}(M)$ is not in $\mathcal{C}_{<n-1}(I, R)$, then there exists $j \in \mathbb{N}_{0}$ such that $\operatorname{Ext}_{R}^{j}\left(R / I, H_{I}^{i}(M)\right) / N_{i, j}$ is not in $\mathcal{C}_{<n-1}(R)$. Let $B_{i, j}=\left\{\mathfrak{q}_{1}, \ldots, \mathfrak{q}_{k}\right\}$ and let $S$ be the multiplicatively closed subset $R \backslash \bigcup_{n=1}^{k} \mathfrak{q}_{n}$. Then applying the same method used above it follows that $S^{-1}\left(\operatorname{Ext}_{R}^{j}\left(R / I, H_{I}^{i}(M)\right) / N_{i, j}\right)$ is a zerodimensional finitely generated $S^{-1} R$-module. Therefore, there exists a finitely generated submodule $U_{i, j} / N_{i, j}$ of the $R$-module $\operatorname{Ext}_{R}^{j}\left(R / I, H_{I}^{i}(M)\right) / N_{i, j}$ such that

$$
S^{-1}\left(\operatorname{Ext}_{R}^{j}\left(R / I, H_{I}^{i}(M)\right) / N_{i, j}\right)=S^{-1}\left(U_{i, j} / N_{i, j}\right) .
$$

But in this situation it is easy to see that $U_{i, j} / N_{i, j}$ is a finitely generated submodule of $\operatorname{Ext}_{R}^{j}\left(R / I, H_{I}^{i}(M)\right) / N_{i, j}$ such that

$$
\operatorname{Supp}\left(\operatorname{Ext}_{R}^{j}\left(R / I, H_{I}^{i}(M)\right) / U_{i, j}\right) \cap B=\emptyset
$$

and hence $\operatorname{Ext}_{R}^{j}\left(R / I, H_{I}^{i}(M)\right) / N_{i, j}$ is in $\mathcal{C}_{<n-1}(R)$, which is a contradiction. Therefore, for each $0 \leq i \leq t$ the $R$-module $H_{I}^{i}(M)$ is in $\mathcal{C}_{<n-1}(I, R)$. Now we are ready to prove that, for each $0 \leq i \leq t$ the $R$-module $H_{I}^{i}(M)$ is in $\mathcal{C}_{<n-2}(I, R)$. By definition for each $0 \leq i \leq t$ and each $j \in \mathbb{N}_{0}$, it suffices to show that there exists a finitely generated submodule $U_{i, j} \subseteq \operatorname{Ext}_{R}^{j}\left(R / I, H_{I}^{i}(M)\right)$ such that $\operatorname{dim}\left(\operatorname{Ext}_{R}^{j}\left(R / I, H_{I}^{i}(M)\right) / U_{i, j}\right)<n-2$. Now in order to complete the proof it is enough to repeat the above argument, for each $0 \leq i \leq t$ and each $j \in \mathbb{N}_{0}$. (Note that just for this time we can use again Lemma 4). 
(ii) The assertion follows immediately from part (i) using Lemma 2.1 and Lemma 2.3.

The following result is the first main result of this paper.

Theorem 2.6. Let $(R, \mathfrak{m})$ be a Noetherian complete local ring, $I$ an ideal of $R$, $t \geq 0$ and $n \geq 2$ be two integers and $M$ be a finitely generated $R$-module such that for each $0 \leq i \leq t$ the $R$-module $H_{I}^{i}(M)$ is in $\mathcal{C}_{<n}(R)$. Then for any finitely generated $R$-module $N$ with support in $V(I)$, each element of the set

$$
\begin{aligned}
\mathfrak{J}= & \left\{\operatorname{Ext}_{R}^{j}\left(N, H_{I}^{i}(M)\right): j \geq 0 \text { and } 0 \leq i \leq t\right\} \\
& \cup\left\{\operatorname{Hom}_{R}\left(N, H_{I}^{t+1}(M)\right), \operatorname{Ext}_{R}^{1}\left(N, H_{I}^{t+1}(M)\right)\right\}
\end{aligned}
$$

is in $\mathcal{C}_{<n-2}(R)$.

Proof. The assertion follows from Theorem 2.5 using Lemma 2.2.

The following results are immediately consequences of Theorem 2.6.

Corollary 2.7. Let $(R, \mathfrak{m})$ be a Noetherian complete local ring, I an ideal of $R$ and $n \geq 2$ be an integer and $M$ be a finitely generated $R$-module such that $\operatorname{dim}(M / I M)=n$. Then for any finitely generated R-module $N$ with support in $V(I)$, each element of the set

$$
\mathfrak{J}=\left\{\operatorname{Ext}_{R}^{j}\left(N, H_{I}^{i}(M)\right): j \geq 0 \text { and } i \geq 0\right\}
$$

is in $\mathcal{C}_{<n-1}(R)$.

Proof. If $\operatorname{dim}(M / I M)=n$ then it is easy to see that the $R$-modules $H_{I}^{i}(M)$ are in $\mathcal{C}_{<n+1}(R)$ for all $i \geq 0$. Therefore the result follows from Theorem 2.6.

Corollary 2.8. Let $(R, \mathfrak{m})$ be a Noetherian complete local ring, $I$ an ideal of $R$, $M$ a finitely generated $R$-module and $n \geq 2$ be an integer. Then for all $i<f_{I}^{n}(M)$ and all $j \geq 0$, the $R$-modules $H_{I}^{i}(M)$ are in $\mathcal{C}_{<n-2}(I, R)$.

Proof. Use [1, Theorem 2.5] and Theorem 2.5.

The following lemma is needed in the proof of our final main result.

Lemma 2.9. Let $R$ be a Noetherian ring, $n \in \mathbb{N}_{0}$ and $M$ be an R-module in $\mathcal{C}_{<n}(R)$. Then the set

$$
\operatorname{Ass}_{R}(M)_{\geq n}:=\left\{\mathfrak{p} \in \operatorname{Ass}_{R}(M): \operatorname{dim}(R / \mathfrak{p}) \geq n\right\}
$$

is finite. 
Proof. By the definition there exists a finitely generated submodule $N$ of $M$ such that $\operatorname{dim}(M / N)<n$. Now from the exact sequence

$$
0 \rightarrow N \rightarrow M \rightarrow M / N \rightarrow 0
$$

it follows that $\operatorname{Ass}_{R}(M)_{\geq n} \subseteq \operatorname{Ass}_{R}(N) \cup \operatorname{Ass}_{R}(M / N)$. But, it is easy to see that

$$
\operatorname{Ass}_{R}(M / N) \cap \operatorname{Ass}_{R}(M)_{\geq n} \subseteq \operatorname{Supp}(M / N) \cap \operatorname{Ass}_{R}(M)_{\geq n}=\emptyset .
$$

So, $\operatorname{Ass}_{R}(M)_{\geq n} \subseteq \operatorname{Ass}_{R}(N)$ and hence $\operatorname{Ass}_{R}(M)_{\geq n}$ is a finite set.

The following result is a generalization of [1, Theorem 2.8].

Theorem 2.10. Let $(R, \mathfrak{m})$ be a Noetherian complete local ring, $I$ an ideal of $R$, $t \geq 0$ and $n \geq 2$ be two integers and $M$ be a finitely generated $R$-module such that for each $0 \leq i \leq t$ the $R$-module $H_{I}^{i}(M)$ is in $\mathcal{C}_{<n}(R)$. Then for any finitely generated $R$-module $N$ with support in $V(I)$, the following statements hold:

(i) For each element $L$ of the set

$$
\begin{aligned}
\mathfrak{J} & =\left\{\operatorname{Ext}_{R}^{j}\left(N, H_{I}^{i}(M)\right): j \geq 0 \text { and } 0 \leq i \leq t\right\} \\
& \cup\left\{\operatorname{Hom}_{R}\left(N, H_{I}^{t+1}(M)\right), \operatorname{Ext}_{R}^{1}\left(N, H_{I}^{t+1}(M)\right)\right\}
\end{aligned}
$$

the set

$$
\operatorname{Ass}_{R}(L)_{\geq n-2}:=\left\{\mathfrak{p} \in \operatorname{Ass}_{R}(L): \operatorname{dim}(R / \mathfrak{p}) \geq n-2\right\}
$$

is finite.

(ii) For each $0 \leq i \leq t+1$, the set

$$
\operatorname{Ass}_{R}\left(H_{I}^{i}(M)\right)_{\geq n-2}=\left\{\mathfrak{p} \in \operatorname{Ass}_{R}\left(H_{I}^{i}(M)\right): \operatorname{dim}(R / \mathfrak{p}) \geq n-2\right\}
$$

is finite.

(iii) The set

$\operatorname{Ass}_{R}\left(\oplus_{i=0}^{f_{I}^{n}(M)} H_{I}^{i}(M)\right)_{\geq n-2}=\left\{\mathfrak{p} \in \operatorname{Ass}_{R}\left(\oplus_{i=0}^{f_{I}^{n}(M)} H_{I}^{i}(M)\right): \operatorname{dim}(R / \mathfrak{p}) \geq n-2\right\}$

is finite.

Proof.

(i) Follows from Theorem 2.6 and Lemma 2.9.

(ii) Follows from (i) using the fact that $\operatorname{Ass}_{R}\left(H_{I}^{i}(M)\right)=\operatorname{Ass}_{R}\left(\operatorname{Hom}_{R}\left(R / I, H_{I}^{i}\right.\right.$ $(M)))$.

(iii) Follows from (ii) using the Corollary 2.8. 
Corollary 2.11. Let $(R, \mathfrak{m})$ be a Noetherian complete local ring, I an ideal of $R$ and $n \geq 2$ be an integer and $M$ be a finitely generated $R$-module such that $\operatorname{dim}(M / I M)=n$. Then for any finitely generated R-module $N$ with support in $V(I)$ and each element $L$ of the set

$$
\mathfrak{J}=\left\{\operatorname{Ext}_{R}^{j}\left(N, H_{I}^{i}(M)\right): j \geq 0 \text { and } i \geq 0\right\}
$$

the set

$$
\operatorname{Ass}_{R}(L)_{\geq n-1}:=\left\{\mathfrak{p} \in \operatorname{Ass}_{R}(L): \operatorname{dim}(R / \mathfrak{p}) \geq n-1\right\}
$$

is finite.

Proof. The assertion follows from Corollary 2.7 and Lemma 2.9.

\section{ACKNOWLEDGMENTS}

The authors are deeply grateful to the referee for his/her careful reading of the paper and valuable suggestions. Also, the authors would like to thank Professor Reza Naghipour for his careful reading of the first draft and many helpful suggestions.

\section{REFERENCES}

1. D. Asadollahi and R. Naghipour, Faltings' local-global principle for the finiteness of local cohomology modules, Comm. Algebra, to appear.

2. K. Bahmanpour, On the category of weakly Laskerian cofinite modules, Math. Scand., to appear.

3. K. Bahmanpour and R. Naghipour, On the cofiniteness of local cohomology modules, Proc. Amer. Math. Soc., 136 (2008), 2359-2363.

4. K. Bahmanpour and R. Naghipour, Associated primes of local cohomology modules and Matlis duality, J. Algebra, 320 (2008), 2632-2641.

5. K. Bahmanpour and R. Naghipour, Cofiniteness of local cohomology modules for ideals of small dimension, J. Algebra, 321 (2009), 1997-2011.

6. K. Bahmanpour, R. Naghipour and M. Sedghi, Minimaxness and cofiniteness properties of local cohomology modules, Comm. Algebra, 41 (2013), 2799-2814.

7. M. P. Brodmann and F. A. Lashgari, A finiteness result for associated primes of local cohomology modules, Proc. Amer. Math. Soc., 128 (2000), 2851-2853.

8. M. P. Brodmann and R. Y. Sharp, Local Cohomology; an Algebraic Introduction with Geometric Applications, Cambridge University Press, Cambridge, 1998.

9. G. Chiriacescu, Cofiniteness of local cohomology modules over regular local rings, Bull. London Math. Soc., 32 (2000), 1-7.

10. D. Delfino, On the cofiniteness of local cohomology modules, Math. Proc. Camb. Phil. Soc., 115 (1994), 79-84. 
11. D. Delfino and T. Marley, Cofinite modules and local cohomology, J. Pure and Appl. Algebra, 121 (1997), 45-52.

12. M. T. Dibaei and S. Yassemi, Associated primes and cofiniteness of local cohomology modules, Manuscripta Math., 117 (2005), 199-205.

13. M. T. Dibaei and S. Yassemi, Finiteness of extension functors of local cohomology modules, Comm. Algebra, 34 (2006), 3097-3101.

14. A. Grothendieck, Local Cohomology, Notes by R. Hartshorne, Lecture Notes in Math., 862, Springer, New York, 1966.

15. A. Grothendieck, Cohomologie Local des Faisceaux Coherents et Théorémes de Lefschetz Locaux et Globaux (SGA2), North-Holland, Amsterdam, 1968.

16. R. Hartshorne, Affine duality and cofiniteness, Invent. Math., 9 (1970), 145-164.

17. C. Huneke and J. Koh, Cofiniteness and vanishing of local cohomology modules, Math. Proc. Camb. Phil. Soc., 110 (1991), 421-429.

18. P. H. Quy, On the finiteness of associated primes of local cohomology modules, Proc. Amer. Math. Soc., 138 (2010), 1965-1968.

19. K.-I. Kawasaki, On the finiteness of Bass numbers of local cohomology modules, Proc. Amer. Math. Soc., 124 (1996), 3275-3279.

20. T. Marley and J. C. Vassilev, Cofiniteness and associated primes of local cohomology modules, J. Algebra, 256 (2002), 180-193.

21. H. Matsumura, Commutative Ring Theory, Cambridge Univ. Press, Cambridge, UK, 1986.

22. K. I. Yoshida, Cofiniteness of local cohomology modules for ideals of dimension one, Nagoya Math. J., 147 (1997), 179-191.

Nemat Abazari and Kamal Bahmanpour

Faculty of Mathematical Sciences

Department of Mathematics

University of Mohaghegh Ardabili

56199-11367, Ardabil

Iran

E-mail: nematabazari@gmail.com

bahmanpour.k@gmail.com 